\title{
Evaluating accredited mHealth applications. An exploratory study
}

\section{Ana Gessa, Amor Jiménez, Pilar Sancha}

Department of Financial Economics, Accounting and Operations Management, University of Huelva, Spain.

\begin{abstract}
Standards and validation practices regarding mobile health apps need to be established to ensure their proper use and integration into medical practice.

This preliminary study aims to conduct a comparative analysis of the entire apps that have been awarded by a Quality Seal to identify significant differences according to the variables analyzed (user, developer, category and consumer ratings) and identified quality attributes.
\end{abstract}

Although the applications analysed are characterised by their heterogeneity, this research found that seven out of 50 remarkable attributes had significant influence on the application evaluation process, according to the recommendations on design, use and assessment of health from AppSaludable. Only some attributes (adaptation of contents, pilot testing, and accessibility and usability) were correlated with some apps'features.

This study can contribute to improving both the processes of validation and quality of medical care of the citizens and in general, the medical practice.

Keywords: health app; Appsaludable; quality seal; eHealth; telecare. 


\section{Introduction}

Digitalisation and technology are present throughout our society and influence all areas of our lives. This development has extended to health care and medical decision-making. The latest advances in mobile communications and technologies have led to the implementation of electronic health records and a huge spectrum of portable wireless devices (mobile health; $\mathrm{m}$-Health) which people can use to transmit, store, process and retrieve real-time and nonreal-time data between patients and medical personnel or between medical personnel (Hansen, Sanchez-Ferro \& Maetzler, 2018; Adibi, 2012).The progressive increase of health apps (h-apps) has led to debate or discussion in recent years to ensure that mobile technology can have a huge impact on healthcare quality and citizens' health. Because the recipients of these apps can be healthcare professionals, medical and nursing students, patients and the general public, the devices can be a valuable tool in health care management (Mosa, Yoo \& Sheets, 2012; Paglialonga, Lugo \& Santoro, 2008).

It is necessary to ensure the quality of these applications, the identified main problems that a health app must address in order to be of quality are security; data protection and reuse of data; possible risks related to misuse; poor regulation; and lack of standards for validation, efficiency and quality (BinDhim, Hawkey \& Trevena, 2015; Huckvale, Prieto, Tilney, Benghozi \& Car, 2015; Martínez-Pérez, De La Torre-Díez \& López-Coronado, 2014).

Medical applications must be regulated and thoroughly reviewed, and carry out a series of measures to improve the development of evidence-based medical applications while maintaining their open character (Buijink, Visser \& Marshall, 2013). There are studies whose aim was to propose a simple, objective, and reliable tool for classifying mobile health apps with a set of criteria and assessing their quality (McMillan, Hickey, Patel \& Mitchell, 2016; BinDhim et al. 2015; Stoyanov, S. R., Hides, L., Kavanagh, D. J., Zelenko, O., Tjondronegoro, D., \& Mani, M. 2015).

In line with all this, there are numerous works that identify the need, on one hand, for a selfcertification model for medical apps (Lewis, 2013) and, on the other hand, to analyse the variables that make them unsafe and of poor quality.

Despite the benefits they offer, better standards and validation practices regarding mobile medical apps need to be established to ensure the proper use and integration of these increasingly sophisticated tools into medical practice. This will help to improve the existing tools and may lead to a better comprehensive m-Health app assessment tool (Nouri, R., Niakan Kalhori, S., Ghazisaeedi, M., Marchand, G., \& Yasini, M. 2018).

Aware of the importance of h-apps in the medical practice, in this paper, we provide an comparative analysis of $\mathrm{h}$-apps with remarkable quality and safety. They have been awarded the AppSaludable Quality Seal. Specific objectives were to: a) Identify the remarkable 
attributes of these apps - both the favourable and those that need improvement - and; b) assess significant relationships among the analysed attributes and selected variables.

The reflections derived from this work can help improve the processes of accreditation of the quality and safety of health applications. These are necessary mechanisms to correct the distortions or bad practices generated by the informational asymmetry inherent in the mobile environment.

\section{Material and Method}

Mixed methods research was selected to achieve the objective of this work. The procedure followed is shown in Figure 1.
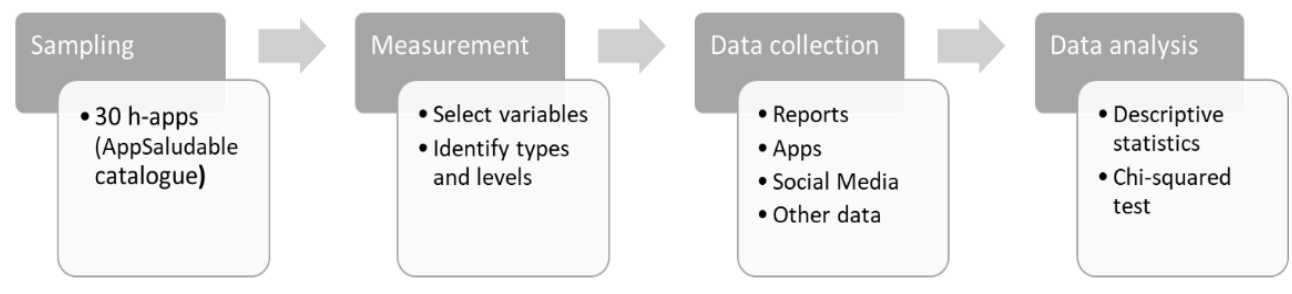

Figure 1. Research methodology.

According to AppSaludable Quality Seal Requeriments (Andalusian Agency for Healthcare Quality, 2012) at the end of April 2019 (http://www.calidadappsalud.com/), 30 health applications were certified. We have analysed all the certified apps. This seal is the first Spanish seal that recognises the quality and safety of health apps. It is free and open to all public or private apps, both Spanish and from other countries. It is a guarantee seal used in order to recognise reliable mobile apps.

This seal is based on 31 recommendations, grouped into four broad categories containing 16 criteria and published in the Guide of Recommendations on Design, Use and Assessment of Health Apps (see Figure 2).

For the analysis of the apps, we collected descriptive information on each app (e.g. price, platform, certification date, etc.) as well as on its technical aspects (e.g. developer, sharing capabilities, etc.). Additional sections collect information on the target user group, as well as information on aspects of the app of interest for the study. These domains may be adapted to include/exclude specific content areas as needed. Next, for each app we extracted the number of downloads and average user rating score. Two variables were identified to evaluate the app: (1) the most frequent remarkable aspects and (2) the issues that need improvement. 


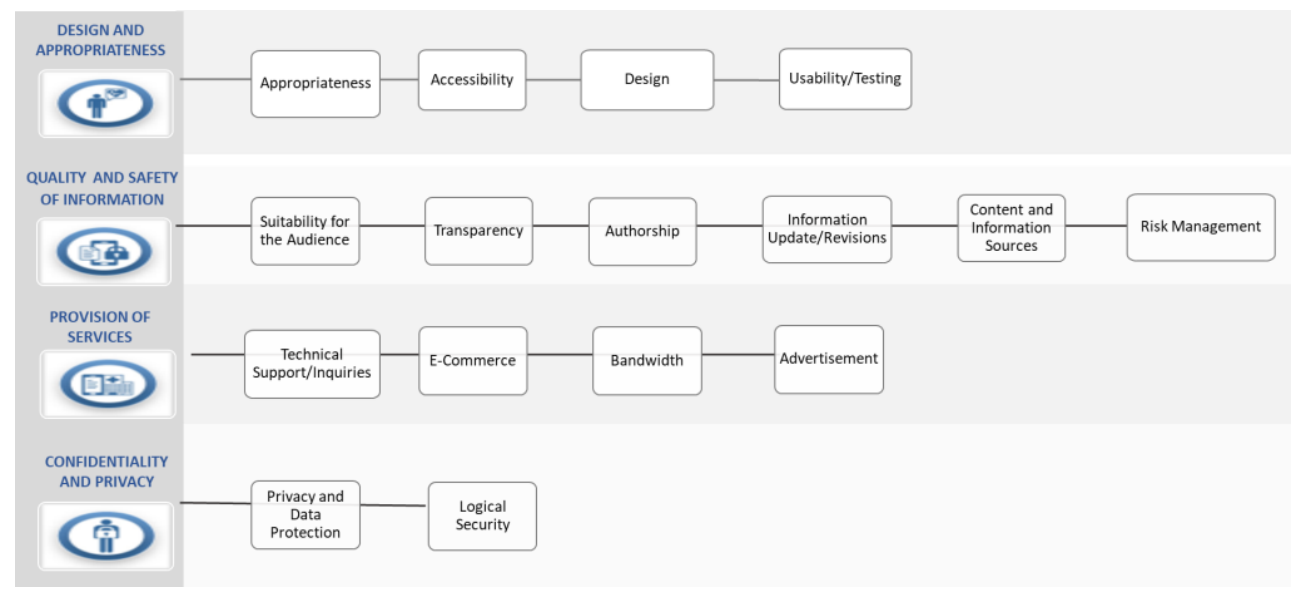

Figure 2. Recommendations on design, use and assessment of health Apps.

Source: http://www.calidadappsalud.com/en/listado-completo-recomendaciones-app-salud/

Data for these variables were taken directly from the Health Apps Catalogue Reports resulting from the evaluation of conformity assessment body. The rest of the variables were obtained by analysing the applications and other specific websites, rankings or social networking, etc. Descriptive statistics were used to summarise h-apps' key characteristics. Some variables, such as platforms, users, main categories and developers, were analysed. In addition, the most frequent remarkable attributes and issues needing attention have been identified using Pareto distribution. Possible relationships between variables were studied through hypothesis test with the Chi square statistic. Analysis results are shown below.

\section{Results and Discussion}

\subsection{Descriptive statistics}

Table 1 summarizes the main characteristics for the 30 mobile health-apps. 
Table 1. Details of $h$-apps $(n=30)$

\begin{tabular}{|c|c|c|c|c|c|}
\hline \multirow{2}{*}{ Characteristics } & \multicolumn{2}{|c|}{ Frequency } & \multirow{2}{*}{ Characteristics } & \multicolumn{2}{|c|}{ Frequency } \\
\hline & No. & $\%$ & & No. & $\%$ \\
\hline Users & & & Seal date & & \\
\hline Patients & 10 & 33.34 & Before 2016 & 15 & 50.00 \\
\hline Professionals & 13 & 43.33 & 2016 & 2 & 6.67 \\
\hline General public & 7 & 23.33 & 2017 & 2 & 6.67 \\
\hline Category & & & 2018 & 9 & 30.00 \\
\hline Monofunction & 21 & 70.00 & 2019 & 2 & 6.66 \\
\hline Multifunction & 9 & 30.00 & Interaction & & \\
\hline Developer & & & Yes & 5 & 16.7 \\
\hline Health sector company & 6 & 20.00 & No & 25 & 83.3 \\
\hline Technological company & 10 & 33.3 & & & \\
\hline Sanitary professional & 10 & 33.3 & & & \\
\hline Particular & 1 & 3.4 & & & \\
\hline Public administration & 3 & 10.00 & & & \\
\hline
\end{tabular}

\subsection{Statistical analysis}

This research shows that seven out of 50 remarkable attributes had significant influence on the application evaluation process according to the AppSaludable recommendations on design, use and assessment of health. The three attributes that are valued the highest are adaptation of the contents to the audience (QA 1), pilot testing (QA 2) and application of universal design principles (QA 9). The four main improvement proposals are editorial commitment to review contents (IP 11), scientific-technical quality control (IP 1), improvement of the accessibility and usability (IP 2) and content selection criteria (IP 18). Results are shown in Figure 3.

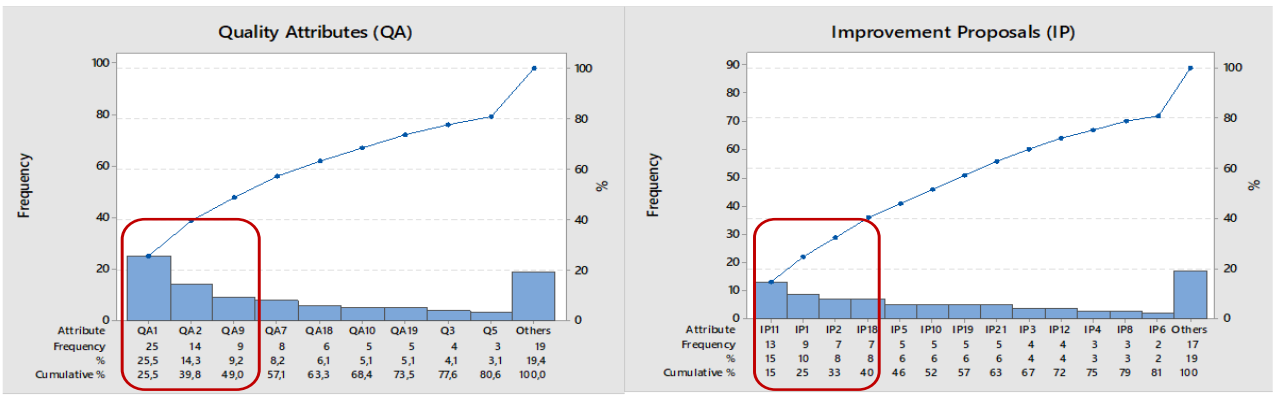

Figure 3. Most frequent quality attributes and improvement proposals of the h-apps.

In order to assess significant relationships among the attributes analysed, a chi-squared test was conducted for the $30 \mathrm{~h}$-apps. Table 2 summarises association test results between the most positive attributes of the h-apps and different selected variables. 
Table 2. Chi-squared test for independence between attributes (QA) and the type of user, category, and developer.

\begin{tabular}{lcccccc}
\hline & \multicolumn{2}{c}{ User } & \multicolumn{2}{c}{ Category } & \multicolumn{2}{c}{ Developer } \\
\cline { 2 - 7 } Attributes & Chi-squared & $\mathbf{p}$ & Chi-squared & $\mathbf{p}$ & Chi-squared & $\mathbf{p}$ \\
\cline { 2 - 7 } QA 1 & 0.733 & 0.693 & 3.981 & 0.046 & 1.298 & 0.9 \\
QA 2 & 3.548 & 0.170 & 0.408 & 0.523 & 9.128 & 0.05 \\
QA 9 & 3.527 & 0.171 & 0.068 & 0.794 & 3.958 & 0.4 \\
\hline
\end{tabular}

Results do not show significant differences in the evaluation of the apps attributable to the type of user. With regards to the category variable, the association is statistically significant $(\mathrm{P}<\alpha=0.05)$ with attribute QA 1; it depends on whether the app has only one function (health and general welfare, medical information, remote monitoring and sensor-based or other) $(64 \%)$ or whether it has more than one function $(36 \%)$.

In the case of the type of developer, the association is statistically significant $(\mathrm{P}<\alpha=0.05)$ with attribute QA 2. The realisation of pilot tests is present in apps created by companies and professionals from the health sector. These represent $65 \%$ of the apps evaluated.

For the issues which need attention, Table 3 shows the results of our statistical analysis.

Table 3. Chi-squared test for independence between improvements proposals (IP) and the type of user, category, and developer.

\begin{tabular}{lcccccc}
\hline & \multicolumn{2}{c}{ User } & \multicolumn{2}{c}{ Category } & \multicolumn{2}{c}{ Developer } \\
\cline { 2 - 7 } Attributes & Chi-squared & $\mathbf{p}$ & Chi-squared & $\mathbf{p}$ & Chi-squared & $\mathbf{p}$ \\
\hline IP 11 & 3.234 & 0.199 & 0.068 & 0.794 & 5.545 & 0.22 \\
IP 1 & 0.784 & 0.676 & 1.190 & 0.275 & 3.721 & 0.45 \\
IP 2 & 0.088 & 0.957 & 0.524 & 0.469 & 9.950 & 0.045 \\
IP 18 & 1.503 & 0.472 & 1.074 & 0.3 & 4.629 & 0.35 \\
\hline
\end{tabular}

A significant relation was found only between accessibility and usability, and the developer. This calls attention to how the developed apps by technology companies and healthcare personnel represent $77 \%$ of the evaluated apps that need to improve that attribute.

Finally, analysis results do not show a relationship between the user experience and 'average user rating score'. However, the apps with the highest user ratings (score $>4$, on a scale of 
1-strongly disagree- to 5-strongly agree-) comprise the highest percentage for the highlight attributes $(86.6 \%, 76.9 \%$ and $85.7 \%$ for attributes QA 1, QA 2 and QA 9 respectively) (see Table 4).

Table 4. Chi-squared test for independence between attributes (QA/IP) and the users' opinion.

\begin{tabular}{|c|c|c|c|c|c|c|c|}
\hline \multicolumn{8}{|c|}{ Users opinion (scale 1-5) } \\
\hline \multicolumn{2}{|l|}{ Attributes } & \multirow{2}{*}{$\frac{\leq 2}{0}$} & \multirow{2}{*}{$\frac{2<x \leq 4}{3}$} & \multirow{2}{*}{$\begin{array}{l}>4 \\
19\end{array}$} & \multirow{2}{*}{$\begin{array}{c}\text { Total } \\
22\end{array}$} & \multirow{3}{*}{$\begin{array}{c}\text { Chi-squared } \\
0.131\end{array}$} & $\mathbf{p}$ \\
\hline \multirow[b]{2}{*}{ QA 1} & Yes & & & & & & \\
\hline & No & 0 & 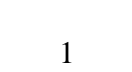 & 4 & 5 & & 0.93 \\
\hline \multirow{2}{*}{ QA 2} & Yes & 0 & 3 & 10 & 13 & \multirow{2}{*}{1.356} & \multirow{2}{*}{0.244} \\
\hline & No & 0 & 1 & 13 & 14 & & \\
\hline \multirow{2}{*}{ QA 9} & Yes & 0 & 1 & 6 & 7 & \multirow{2}{*}{0.002} & \multirow{2}{*}{0.963} \\
\hline & No & 0 & 3 & 17 & 20 & & \\
\hline \multirow{2}{*}{ IP 11} & Yes & 0 & 2 & 2 & 4 & \multirow{2}{*}{0.934} & \multirow{2}{*}{0.334} \\
\hline & No & 0 & 6 & 17 & 23 & & \\
\hline \multirow{2}{*}{ IP 1} & Yes & 0 & 1 & 5 & 6 & \multirow{2}{*}{0.021} & \multirow{2}{*}{0.99} \\
\hline & No & 0 & 3 & 18 & 21 & & \\
\hline \multirow{2}{*}{ IP 2} & Yes & 0 & 2 & 10 & 12 & \multirow{2}{*}{0.059} & \multirow{2}{*}{0.809} \\
\hline & No & 0 & 2 & 13 & 15 & & \\
\hline \multirow{2}{*}{ IP 18} & Yes & 0 & 1 & 5 & 6 & \multirow{2}{*}{0.021} & \multirow{2}{*}{0.99} \\
\hline & No & 0 & 3 & 18 & 21 & & \\
\hline
\end{tabular}

\section{Conclusions}

There is no doubt that m-Health is a key factor in the challenge of moving towards more sustainable health, improving efficiency and effectiveness, reducing costs and meeting the main needs of our society. Therefore, in this context, the research reveals the suitability of an assessment tool with a wide scope of application, identifying the most frequent positive and negative h-apps attributes.

The results of this work show that the certification model is in the growth state. Administration is concerned about the correct use of these applications in evolving toward a state of maturity. However, this will only be possible if the weaknesses detected in this study are corrected. In conclusion, we would emphasise the following issues: 
- It should be noted the lack of integration in a larger project (national, European or international) that supplies a wide coverage and reliability of the seal application criteria.

- This research has detected that the seal must demand a higher level in technical qualities, content and security. We highlight the recommendations concerning accessibility and usability requirements as pointed out in the $44 \%$ of the samples analysed. In particular, this attribute performed significantly with the variable “developer" (see Table 3).

- The wide heterogeneity in assessment criteria for $\mathrm{m}$-Health requires a redefinition of the meanings of each criterion.

- Because of the lack of a significant relationship between remarkable attributes and average user rating score, perhaps a re-examination of the seal evaluation process would be useful.

These initiatives definitely are welcome in the field of medicine, as demonstrated by the 80 apps waiting to obtain the AppSaludable seal. Obviously, it can contribute to improving both the processes of validation and the quality of citizens' medical care.

\section{References}

Adibi, S. (2012). Link technologies and BlackBerry mobile health (mHealth) solutions: a review. IEEE Transactions on Information Technology in Biomedicine, 16(4), 586-597. http://doi.org/10.1109/TITB.2012.2191295

BinDhim, N. F., Hawkey, A., \& Trevena, L. (2015). A systematic review of quality assessment methods for smartphone health apps. Telemedicine and e-Health, 21(2), 97104. http://dx.doi.org/10.1136/bmjinnov-2014-000019

BinDhim, N. F., \& Trevena, L. (2015). Health-related smartphone apps: regulations, safety, $\begin{array}{llll}\text { privacy and } \quad \text { quality. BMJ } & \text { 43-45. }\end{array}$ https://doi.org/10.1089/tmj.2014.0088

Buijink, A. W. G., Visser, B. J., \& Marshall, L. (2013). Medical apps for smartphones: lack of evidence undermines quality and safety. Evidence-based medicine, 18(3), 90. http://dx.doi.org/10.1136/eb-2012-100885

Hansen, C., Sanchez-Ferro, A., \& Maetzler, W. (2018). How mobile health technology and electronic health records will change care of patients with Parkinson's disease. Journal of Parkinson's disease, 8(s1), S41-S45. http://doi.org/10.3233/JPD-181498

Huckvale, K., Prieto, J. T., Tilney, M., Benghozi, P. J., \& Car, J. (2015). Unaddressed privacy risks in accredited health and wellness apps: a cross-sectional systematic assessment. BMC medicine, 13(1), 214. https://doi.org/10.1186/s12916-015-0444-y

Lewis, T. L. (2013). A systematic self-certification model for mobile medical apps. $J$ Med Internet Res, 15(4), e89. https://doi.org/10.2196/jmir.2446 
Martínez-Pérez, B., De La Torre-Díez, I., \& López-Coronado, M. (2015). Privacy and security in mobile health apps: a review and recommendations. Journal of medical systems, 39(1), 181. https://doi.org/10.1007/s10916-014-0181-3

McMillan, B., Hickey, E., Patel, M. G., \& Mitchell, C. (2016). Quality assessment of a sample of mobile app-based health behavior change interventions using a tool based on the National Institute of Health and Care Excellence behavior change guidance. Patient education and counseling, 99(3), 429-435. https://doi.org/10.1016/j.pec.2015.10.023

Mosa, A. S. M., Yoo, I., \& Sheets, L. (2012). A systematic review of healthcare applications for smartphones. BMC medical informatics and decision making, 12(1), 67. https://doi.org/10.1186/1472-6947-12-67

Nouri, R., Niakan Kalhori, S., Ghazisaeedi, M., Marchand, G., \& Yasini, M. (2018). Criteria for assessing the quality of mHealth apps: a systematic review. Journal of the American Medical Informatics Association, 25(8), 1089-1098.

https://doi.org/10.1093/jamia/ocy050

Paglialonga, A., Lugo, A., \& Santoro, E. (2018). An overview on the emerging area of identification, characterization, and assessment of health apps. Journal of biomedical informatics, 83, 97-102. http://doi.org/10.1016/j.jbi.2018.05.017

Stoyanov, S. R., Hides, L., Kavanagh, D. J., Zelenko, O., Tjondronegoro, D., \& Mani, M. (2015). Mobile app rating scale: a new tool for assessing the quality of health mobile apps. JMIR mHealth and uHealth, 3(1), e27. https://doi.org/10.2196/mhealth.3422 